\title{
Atmospheric boundary layer height disambiguation using synergistic remote sensing observations: case examples from VORTEX-SE
}

Rocadenbosch, Francesc, Tanamachi, Robin, Araujo da Silva, Marcos, Villalonga, Joan, Frasier, Stephen, et al.

Francesc Rocadenbosch, Robin L. Tanamachi, Marcos P. Araujo da Silva, Joan Villalonga, Stephen J. Frasier, David D. Turner, "Atmospheric boundary layer height disambiguation using synergistic remote sensing observations: case examples from VORTEX-SE," Proc. SPIE 11531, Remote Sensing of Clouds and the Atmosphere XXV, 115310L (20 September 2020); doi: $10.1117 / 12.2576093$

Copyright 2020 Society of Photo Optical Instrumentation Engineers. One print or electronic copy may be made for personal use only. Systematic electronic or print reproduction and distribution, duplication of any material in this paper for a fee or for commercial purposes, or modification of the content of the paper are prohibited.

http://dx.doi.org/10.1117/12.2576093 


\title{
Atmospheric Boundary Layer Height Disambiguation using Synergistic Remote Sensing Observations: Case Examples from VORTEX-SE
}

\author{
Francesc Rocadenbosch ${ }^{1,2}$, Robin L. Tanamachi ${ }^{3}$, Marcos P. Araujo da Silva ${ }^{1}$, Joan \\ Villalonga $^{1}$, Stephen J. Frasier ${ }^{4}$, and David D. Turner ${ }^{5}$ \\ ${ }^{1}$ CommSensLab-UPC, Department of Signal Theory and Communications, Universitat \\ Politècnica de Catalunya (UPC), C/ Jordi Girona, 1-3, E-08034, Barcelona, Spain. \\ ${ }^{2}$ Institut d'Estudis Espacials de Catalunya (Institute of Space Studies of Catalonia, IEEC), \\ Barcelona, Spain, E-08034 Barcelona, Spain \\ ${ }^{3}$ Dept. of Earth, Atmospheric, and Planetary Sciences, Purdue University, 550 West Stadium \\ Avenue, West Lafayette, IN 47906 (USA). \\ ${ }^{4}$ Microwave Remote Sensing Laboratory, University of Massachusetts, 151 Holdsworth Way, \\ Suite 113, Amherst, MA 01003 (USA). \\ ${ }^{5}$ Global Systems Laboratory / Earth System Research Laboratories / NOAA, 325 Broadway, \\ Boulder, CO 80305-3337 (USA).
}

\begin{abstract}
Synergistic remote sensing of the atmosphere, combined with adaptive techniques, offers unprecedented opportunities to characterise the evolution of key atmospheric features such as the Atmospheric Boundary Layer (ABL). Using long-duration, high-resolution, profiling observations from active and passive ground-based remote sensing systems during the Verification of the Origins of Rotation in Tornadoes Experiment-Southeast (VORTEX-SE) 2017 field campaign, an attempt is made to characterise ABL development over distinct regions that are well known for their relatively high tornado frequency. In this study, observing systems include an S-band radar, Vaisala CL-31 ceilometer, Doppler Wind lidar (DWL) and radiometric observations from the Collaborative Lower Atmosphere Mobile Profiling System (CLAMPS). In this work, ABL height (ABLH) tracking over the diurnal cycle, and - up to a point - its disambiguation over selected non-precipitating case examples, are attempted. Different observational sets are used, namely, radar reflectivity observations assimilated into a Kalman filter, DWL profiles of the vertical velocity, and virtual potential temperature profiles, as well as radiosoundings and cloudbase reference information collected during Intensive Observation Periods (IOP) carried out in VORTEX-SE, Alabama during 2017. Limitations and advantages of each system are discussed.
\end{abstract}

Keywords: atmospheric boundary layer, S-band radar, ceilometer, Doppler lidar, adaptive filtering, estimation.

\section{INTRODUCTION}

The lowest layer of the atmosphere, the one most affected by interactions with the Earth's surface, is called the atmospheric boundary layer (ABL). ${ }^{1}$ The ABL is characterised by large spatial and temporal variability. ${ }^{2}$ During fair weather days over land, the ABL behaviour is generally described by a diurnal cycle with three main structural components:

1. The convective boundary layer (CBL), which develops during the daytime as a result of turbulence generated by solar heating of the land surface.

Further author information: (Send correspondence to F.R. and R.T.)

F.R.: E-mail: roca@tsc.upc.edu, Telephone: +34 934054114

R.T.: E-mail: rtanamachi@purdue.edu, Telephone: +1 (765) 496-2866

Remote Sensing of Clouds and the Atmosphere XXV, edited by Adolfo Comerón, Evgueni I. Kassianov, Klaus Schäfer, Richard H. Picard, Konradin Weber, Upendra N. Singh, Proc. of SPIE Vol. 11531,

115310L · C 2020 SPIE · CCC code: 0277-786X/20/\$21 - doi: 10.1117/12.2576093 
2. The residual layer (RL), which forms around half an hour before local sunset as turbulence intensity decays, maintaining residual moisture and heat character of the CBL; and

3. The stable boundary layer (SBL), which is formed by the cold air yielded in response to the surface cooling throughout the night progress. The SBL is normally characterized by static stable air that leads to suppress the turbulence. ${ }^{1,2}$

The Verification of the Origins of Rotation in Tornadoes Experiment-Southeast (VORTEX-SE) is a project devoted to understanding the environmental factors related to the formation and intensity of tornadoes in the southeastern United States. ${ }^{3}$ Moreover, the experiment is designed to account for the associated societal impacts; evaluating, for example, the optimal procedures to communicate the tornado forecast and risks to the public. ${ }^{3}$ From the meteorological point of view, one of the main objectives of the project is the intensive monitoring of the ABL evolution with high spatio-temporal resolution, aiming to achieve a more comprehensive knowledge about the tornadogenesis potential of the storm's environment. ${ }^{4}$

In this study, we investigate the ABL development in VORTEX-SE domain by means of a synergistic remote sensing approach, in which multiple independent estimates of the ABL height (ABLH) from distinct instruments are retrieved. We use observational profiles of atmospheric reflectivity and vertical (air) velocity (VV) measured by an S-band radar and a Doppler Wind Lidar (DWL), respectively, as well as two independent estimations of virtual potential temperature (VPT), retrieved from the Atmospheric Emitted Radiance Interferometer (AERI) and radiosondes (RS).

$S$-band radars (with wavelength $10 \mathrm{~cm}$ ) have been used to monitor the ABL for more than forty years..$^{5-7}$ They measure VV via a combination of Rayleigh and Bragg scattering. The $D W L$ became more common in the last two decades as wind energy industry applications spurred its evolution. ${ }^{8}$ The DWL has been applied successfully for CBL characterization, because the VV-DWL can clearly detect turbulence within the convective updrafts and downdrafts during convective periods. ${ }^{9}$

The AERI is a passive spectrometer that measures the downwelling infrared radiance emitted by the atmosphere, which is a physical parameter sensitive to the atmospheric vertical thermodynamic structure. Thus, the AERI observational radiance is useful to retrieve profiles of temperature and humidity at relatively high temporal resolution (about every minute). ${ }^{10}$ The RS is a standard reference instrument used to obtain information about the state of the atmosphere, consisting of a miniaturized, expendable, balloon borne meteorological sensor package. Historically, RS have been used since the 1930s to deliver accurate profiles of pressure, temperature, water vapour and wind ${ }^{11}$ for operational forecasting purposes. However, each launch entails considerable expense, and therefore the number of samples is constrained by the frequency of launches (on the order of hourly).

In this study, the different estimates of the ABLH derived from these four instruments are compared for two different scenarios: (i) an ABL diurnal cycle on a cloud-free day, and (ii) a period characterized by low clouds. During VORTEX-SE, RS data is usually only available for the second scenario. The present manuscript is paired with a companion paper, ${ }^{12}$ also aimed at CBL monitoring in the context of the VORTEX-SE 2017. This study is organized as follows: Sect. 2 describes the instruments and the ABLH retrieval methods, Sect. 3 presents the two ABLH monitoring scenarios, and, Sect. 4 gives concluding remarks.

\section{INSTRUMENTS AND METHODS}

Next, we summarize the instruments and ABLH retrieval methods used (see Ref. 12 for details). Data were collected during the VORTEX-SE field campaign from 10 March to 1 May 2017. ${ }^{4}$ For this campaign, the instruments used in this study were collocated at Scottsboro, Alabama municipal airport $\left(34.68728{ }^{\circ} \mathrm{N}, 86.00508\right.$ $\left.{ }^{\circ} \mathrm{W}\right)$. Two systems were deployed to monitor the ABL cycle: the University of Massachusetts S-band radar (hereinafter UMass S-band radar), ${ }^{4,5}$ which is a transportable, vertically-pointing FMCW radar mounted on a truck, and the Collaborative Lower Atmospheric Mobile Profiling System (CLAMPS-2 ${ }^{13}$ ), which is mobile unit in a trailer comprising a Halo Stream Line XR DWL and the AERI v4 MP-3000A. Both systems were capable of collecting, processing, and uploading their data in near-real time. During VORTEX-SE 2017 Intenstive Observing Periods (IOPs), Vaisala RS-41 radiosondes were launched from Scottsboro airport at roughly hourly intervals. A Vaisala CL31 ceilometer, collocated with the UMass S-band radar, measured cloud base height (CBH). The main specifications of the instruments are summarized in Tab. 1. 
Taula 1. Main specifications of the instruments. (HPBW) Half Power Beam Width. (1) VAD velocity-azimuth display scan. (2) Products retrieved from AERIoe. (3) Height-dependent spatial resolution: $10 \mathrm{~m}$ adjacent to the surface and $300 \mathrm{~m}$ at $3 \mathrm{~km}^{13}$ (Reproduced with permission from Ref. 12).

\begin{tabular}{|c|c|c|c|c|c|}
\hline Instrument & $\begin{array}{c}\text { Measured } \\
\text { Parameters }\end{array}$ & Pointing & Wavelength & $\begin{array}{l}\text { Raw spatial } \\
\text { resolution }\end{array}$ & $\begin{array}{l}\text { Raw temporal } \\
\text { resolution }\end{array}$ \\
\hline $\begin{array}{l}\text { UMass } \\
\text { S-band radar }\end{array}$ & $\begin{array}{c}\text { Volume } \\
\text { reflectivity } \\
{\left[\mathrm{m}^{-1}\right]}\end{array}$ & $\begin{array}{c}3 \mathrm{deg} \\
(\mathrm{HPBW})\end{array}$ & $\begin{array}{c}10 \mathrm{~cm} \\
(\mathrm{FMCW})\end{array}$ & $5 \mathrm{~m}$ & $16.1 \mathrm{~s}$ \\
\hline $\begin{array}{l}\text { DWL (Halo } \\
\text { Stream Line } \\
\text { XR) }\end{array}$ & $\begin{array}{l}\text { Vertical air } \\
\text { velocity }[\mathrm{m} \\
\left.\mathrm{s}^{-1}\right]\end{array}$ & $\begin{array}{c}\mathrm{VAD}^{(1)} \\
\text { Zenith } \\
\text { sampling }\end{array}$ & $1.5 \mu \mathrm{m}$ & $26 \mathrm{~m}$ & $\begin{array}{l}3 \mathrm{~min} \\
1 \mathrm{~Hz}\end{array}$ \\
\hline $\begin{array}{l}\text { AERI (v4 } \\
\text { MP-3000A) }\end{array}$ & $\begin{array}{c}\text { AERIoe }{ }^{(2)} \text { : } \\
\text { Temperature } \\
{\left[{ }^{\circ} \mathrm{C}\right]} \\
\text { Water Vapor } \\
{\left[\mathrm{g} \mathrm{kg}^{-1}\right]}\end{array}$ & Vertically & $\begin{array}{c}3.3-18.2 \\
\mu \mathrm{m}\end{array}$ & $\begin{array}{c}10-300 \\
\mathrm{~m}^{(3)}\end{array}$ & $5 \min$ \\
\hline
\end{tabular}

\subsection{Instruments and ABLH retrievals}

\subsubsection{Radar ABLH retrieval}

The UMass S-band radar is a Frequency Modulated Continuous Wave (FMCW), $2.92 \mathrm{GHz}$, pulse-compression radar that has been used to retrieve vertical profiles of the atmospheric refractive-index since 1999. This radar is equipped with a $250 \mathrm{~W}$ solid state amplifier transmitter, and two 2.4-m parabolic dish antennas, both with 34 dB gain. ${ }^{5}$ UMass S-band easily detects enhanced Bragg scatter at the turbulent interface between the CBL and the free troposphere (FT) above. ${ }^{4}$ The ABLH was estimated from UMass S-band data by applying an extended Kalman filter (EKF) to the sequence of "clean" reflectivity profiles, i.e., removing non-meteorological echoes from insects, birds, and aircraft traffic in the raw radar reflectivity. ${ }^{14}$

We use the radar-EKF technique described in Ref. 15, which is also validated for lidar backscatter returns. ${ }^{16}$ In this approach, an erf-like function parameterization models the abrupt CBL-to-FT) transition and automatically retrieves the ABLH by tracking the inflection point of the CBL-to-FT parameterization. The EKF achieves an optimal ABLH estimation by recursively relating the present radar reflectivity profile to past estimates of ABLH, measurement-noise covariance, and by using a state-vector model roughly describing the time transitions of CBL-to-FT parameterization. The EKF also provides the uncertainty of the ABLH estimated by means of an a posteriori error covariance matrix. ${ }^{15}$ Fig. 1 shows two examples of the radar-EKF retrieval.

\subsubsection{DWL ABLH retrieval}

The VV profiles are retrieved by means of the Velocity-Azimuth Display (VAD ${ }^{17,18}$ ) algorithm, using as input the Halo DWL air velocity data measured along multiple conical lines of sight. During the VORTEX-SE 2017 field campaign, the Halo DWL was configured to perform the VAD scan by sampling eight fixed azimuth angles at $60^{\circ}$ elevation at Low-temporal Resolution (LR) of 3 min. ${ }^{13}$ During VORTEX-SE 2017 IOPs, High-temporal Resolution $(\mathrm{HR})(\Delta t \approx 0.7 \mathrm{~s}) \mathrm{VV}$ measurements taken by the DWL pointing vertically are sometimes also available.

Using the VV profiles, the ABLH is estimated by applying a threshold to the VV standard deviation (VVSTD), $\sigma_{t h}=0.15 \mathrm{~m} \mathrm{~s}^{-1}$, thereby defining the ABLH as the first height where the VVSTD decreases below this threshold. ${ }^{8}$ In the case of HR VV data, the computation of the VVSTD must be done using the noise removing techniques described in Ref. 19. We also use a higher VVSTD threshold $\left(\sigma_{t h}=0.2 \mathrm{~m} \mathrm{~s}^{-120}\right)$ for the $\mathrm{HR}$ data because the VAD technique provided smoothed values of VV. In order to quantify the uncertainty of this estimation, we perturb the VVSTD threshold, $\sigma_{t h} \pm 0.05 \mathrm{~m} \mathrm{~s}^{-1}$, yielding two new ABLH estimates in response, denoted $z_{\sigma_{t h \mp 0.05}}$, which correspond to the upper and lower bounds of the estimated ABLH. Because 

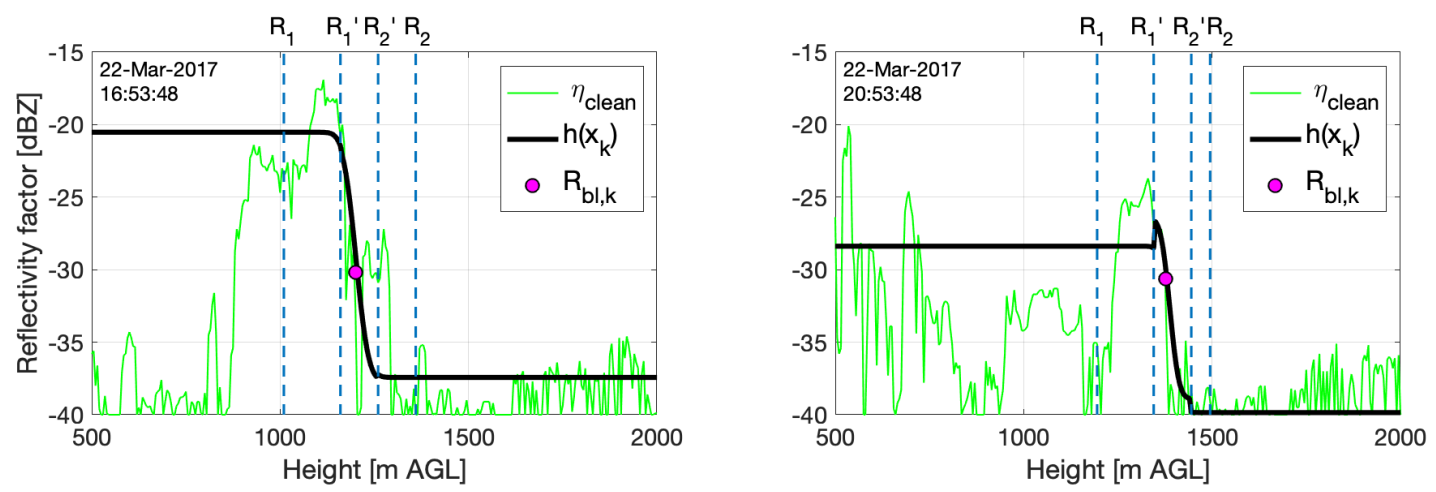

Figura 1. Radar-EKF retrieval examples from 22 March 2017 showing the growth of the CBL. (a) 1653 UTC (1153 LT). (b) 2053 UTC (1553 LT). CBL-to-FT parameterization (erf-like function; black line) fitted to the "clean" reflectivity profile (green line), overlaid with estimated ABLH (magenta dot). $R_{1}$ and $R_{2}$ delimit the height ranges considered in the EKF erf model fitting. $R_{1}^{\prime}$ and $R_{2}^{\prime}$ denote the approximate start and end ranges of the erf transition, which approximate the span of the EZ.

this method measures the turbulence intensity though the $\mathrm{VV}$, it has the advantage of being less sensitive to residual layers which may lead to incorrect ABLH estimates. ${ }^{9}$

In Fig 2, we compare the ABLH estimates based on HR and LR VV data during the convective regime of 16 April 2017. During most of the convective period (1500 to 2000 UTC), the HR and LR estimates agree to within their respective uncertainties (Fig. 2a). From 2000 UTC onwards, however, the performance of the ABLH estimates from LR data deteriorate. We speculate that, after local noon (i.e., 1700 UTC), turbulence peaks and starts to decrease. As a result, the estimate of the VVSTD made from LR data becomes less accurate, which leads to incorrect DWL-based ABLH estimate. Another factor that likely contributes to the superiority of the ALBH estimates from the HR DWL data is the smaller uncertainty associated with the HR data. This is best evidenced in Fig. 2b, where the difference between the estimated ABLH for a given threshold $\left(\sigma_{t h}=0.2 \mathrm{~m} \mathrm{~s}^{-1}\right.$, $\left.\mathrm{HR} ; \sigma_{t h}=0.15 \mathrm{~m} \mathrm{~s}^{-1}, \mathrm{LR}\right)$ and the estimated ABLH resulting from perturbing these thresholds by $\pm 0.5 \mathrm{~m}$ $\mathrm{s}^{-1}$ is plotted. Finally, in Fig. 2c, we compare the shape of the vertical profiles of the VVSTD $\left(\sigma_{w}\right)$ with the semi-theoretical model proposed by Ref. 21. It is evident that the HR VVSTD profile is in closer agreement with the model than the LR VVSTD profile. This result justifies selection of a lower threshold $\left(\sigma_{t h}\right)$ for the LR data in order to obtain similar quality ABLH estimates using both temporal resolutions.

We can conclude that HR DWL data provides more reliable ABLH estimates due to its lower sensitivity to the selected VVSTD threshold as well as the closer agreement with the model. In the cases in which LR data are available and HR data are not, reducing the threshold $\sigma_{t h}$ can yield similar quality ABLH estimates to HR data over most of the CBL. In this project, however, our access to DWL HR data was restricted to a very few particular days. For this reason, hereafter, the DWL data used will have low temporal resolution.

\subsubsection{AERI}

In the case of the AERI, the spectral downwelling infrared radiance is used as input for the AERI Optimal Estimation algorithm (AERIoe), which is an iterative Gauss-Newton algorithm that retrieves profiles of temperature and humidity in both clear-sky and cloudy cases. ${ }^{10}$ Additionally, the AERIoe algorithm provides liquid water cloud properties and a covariance matrix for each of the obtained profiles. The AERIoe products are retrieved on a vertical grid that decreases exponentially with height, from $10 \mathrm{~m}$ adjacent to the surface to $300 \mathrm{~m}$ at $3 \mathrm{~km}$ AGL. ${ }^{13}$

\subsubsection{VPT ABLH retrieval}

The thermodynamic profiles derived from AERIoe and RS were used to independently retrieve profiles of virtual potential temperature (VPT), denoted $\theta_{v}$ in meteorological literature. Some of the RS profiles do not provide accurate thermodynamic information at ground level, and hence, they do not allow the application of the parcel 
(a)

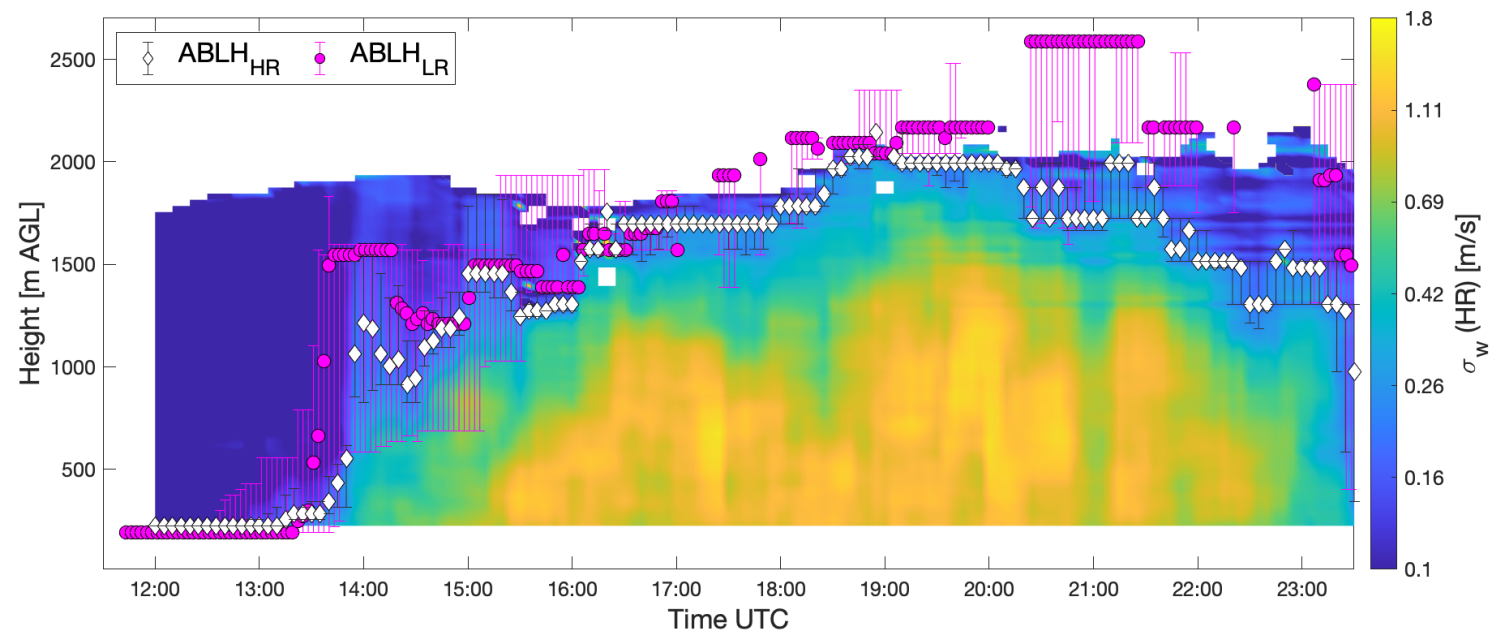

(b)

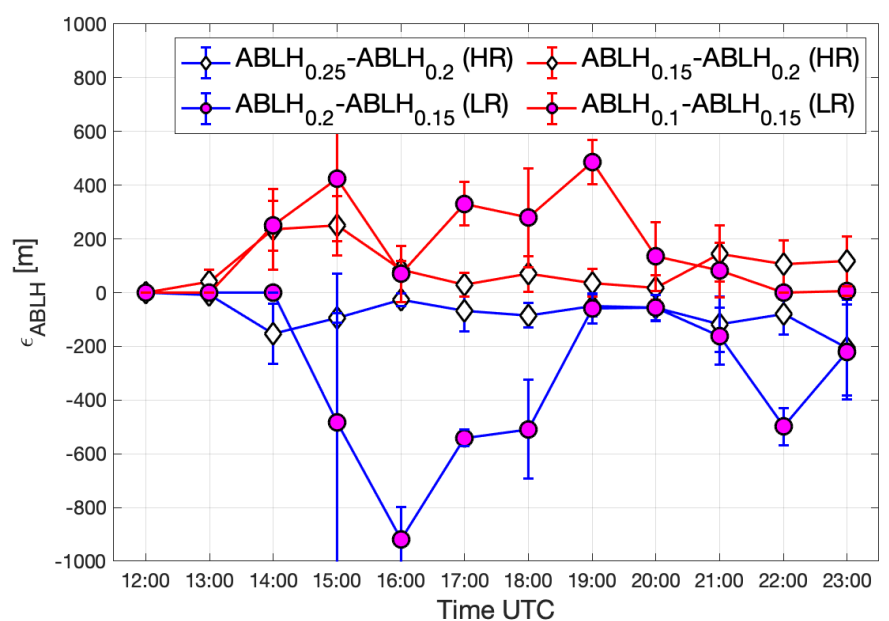

(c)

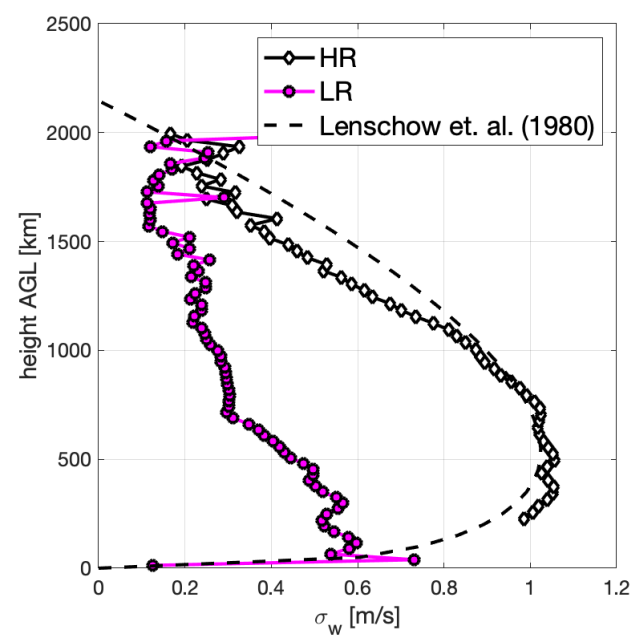

Figura 2. Comparison between DWL ABLH estimates using high (HR) and low temporal resolution (LR) VV data from 16 April 2017. (a) (Colorplot) VVSTD computed from the HR data, overlaid with ABLH estimates derived from HR and LR data (white diamonds and magenta circles, respectively). ABLH estimates and associated uncertainties are computed using the threshold method explained in Sect. $2.1 .2\left(\sigma_{t h}=0.2 \mathrm{~m} \mathrm{~s}^{-1}\right.$, $\mathrm{HR} ; \sigma_{t h}=0.15 \mathrm{~m} \mathrm{~s}^{-1}$, LR). (b) ABLH threshold sensitivity computed as the difference between the ABLH estimate given $\sigma_{t h}$, and corresponding lower and upper bounds computed by perturbing $\sigma_{t h} \pm 0.05 \mathrm{~m} / \mathrm{s}$. White diamonds (magenta circles) correspond to HR (LR) data; the corresponding upper (lower) bounds are drawn as colored red (blue) lines. (c) Average VVSTD for the period 1500-2000 UTC as function of height, computed from HR and LR data (white diamonds and magenta circles, respectively). The black-dashed line stands for the Lenschow reference model (Ref. 21). 
method (PM). For this reason, we opted for the gradient method (GM), which defines the ABLH as the height corresponding to the maximum gradient of the VPT. ${ }^{1}$ The RS provide data with height resolution of 2-8 $\mathrm{m}$. Considered in this study are 55 launches spanning the whole 2017 field campaign, distributed over 10 different days. In contrast to RS VPT profiles, AERIoe vertical profiles have coarser, height-dependent vertical resolution. To obtain the optimal ABLH retrievals from the AERIoe VPT profiles, which have different shapes as those from the RS, the PM and GM are adapted (discussed below) and applied during the CBL and the SBL periods, respectively.

The PM defines the ABLH as the altitude where VPT has the same value as the surface VPT. ${ }^{22}$ Direct application of the PM to individual AERIoe VPT-derived profiles may often lead to incorrect ABLH estimates, because of the coarser height resolution of the VPT profiles with increasing height. This effect is more pronounced above $1 \mathrm{~km}$ AGL. To alleviate this issue, the PM method is applied over 100 different VPT profiles obtained by perturbing randomly the current VPT profile within the uncertainty range provided by the a posteriori error covariance matrix given by the AERIoe at the same time. ${ }^{10}$ The 100 ABLH estimates computed are averaged, thus retrieving the ABLH with an uncertainty of $\pm 1 \sigma$, where $\sigma$ is the standard deviation of the averaged-sample distribution. Fig. 3b-c show some examples of AERI retrieved VPT profiles and the estimated ABLH.

In SBL periods, we follow the method proposed by Ref. 1, Eq. 12.1.3d, although the ABLH is computed in a different way. In this method, the profile of the VPT is modelled by a polynomial that passes through the surface value and reaches zero gradient at the top of the SBL model, similar to the VPT profiles shown in Fig. 3a. We estimate the ABLH (i.e., the SBL top) as the first cornerpoint where the VPT gradient changes from positive to negative (notice that in Fig. 3a, 0900 UTC profile, the ABLH is retrieved at $550 \mathrm{~m}$ and not $250 \mathrm{~m}$, the latter one corresponding to a positive-to-positive-gradient cornerpoint). In some VPT profiles, this positive to negative gradient transition does not occur, then we estimate the ABLH at the height with minimum gradient.

(a)

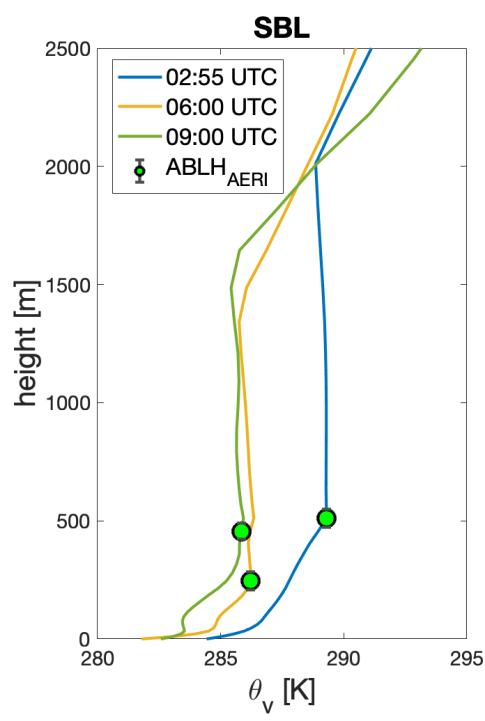

(b)

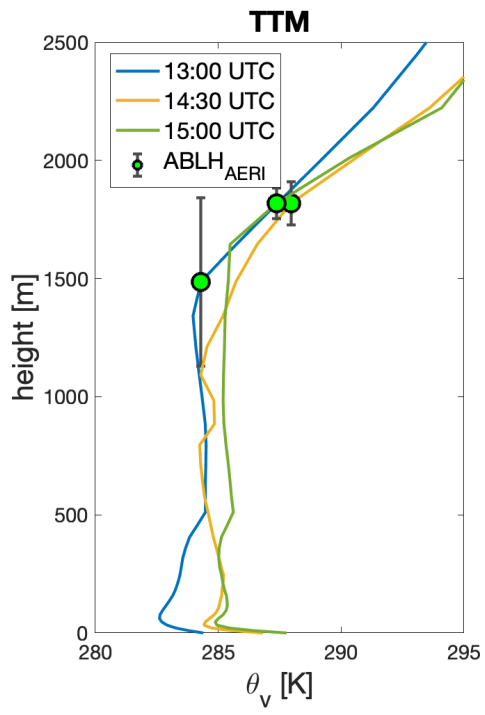

(c)

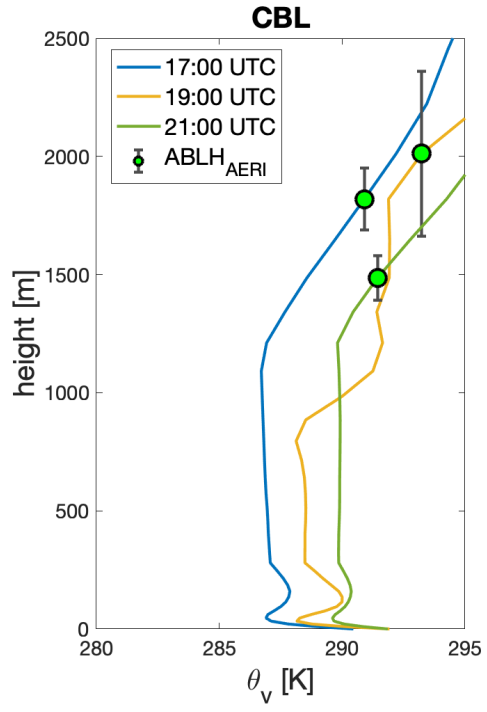

Figura 3. Virtual potential temperature profiles retrieved from the AERIoe and corresponding ABLH estimates at different phases of the diurnal ABL cycle on 7 April 2017): (a) Stable Boundary Layer (SBL) period, (b) Transition Time-Morning (TTM) period, and (c) Convective Boundary Layer (CBL) period. Green dots represent the ABLH estimated using local gradient extrema.

\subsection{Hourly-averaged ABLH estimation and uncertainty}

ABLH estimates are first computed at the respective temporal resolutions of the different instrument-method combinations considered (Sect. 2.1). In order to inter-compare all the results in a meaningful statistical sense, 
each set of estimates is standardized to hourly averages ABLH computed via Maximum-Likelihood average, ${ }^{23}$

$$
A B L H_{I}\left(t_{h}\right)=\frac{\sum_{i}\left(z_{i} / \sigma_{i}^{2}\right)}{\sum_{i}\left(1 / \sigma_{i}^{2}\right)},
$$

where $I$ stands for "intrument" $\left(I=\mathrm{AERI}\right.$, DWL, S-band radar), $t_{h}$ is the hourly time (in UTC), $z_{i}$ is the instantaneous ABLH estimated at time $t_{i}$, subindex $i$ stands for $t_{i} \in\left[t_{h}-30 \mathrm{~min}, \ldots, t_{h}+30 \mathrm{~min}\right]$, and $\sigma_{i}$ is the ABLH associated uncertainty of the ABLH estimation at the time $t_{i}$. The Maximum-Likelihood estimate provides a trustworthy estimation of the ABLH because samples with high uncertainties are given a low weight in the weighted mean, therefore reducing weights of outliers.

For each hourly estimate, $A B L H_{i}\left(I=\mathrm{AERI}\right.$, DWL, UMass S-band radar) the associated uncertainty, $\sigma_{I}$, is computed as:

$$
\sigma_{I}=\sqrt{\sigma_{I 1}^{2}+\sigma_{I 2}^{2}}
$$

where $\sigma_{I 1}$ is the atmospheric variability of the estimated $A B L H$ as measured by instrument $I$,

$$
\sigma_{I 1}=\operatorname{std}\left(z_{i}\right),
$$

and $\sigma_{I 2}$ is the uncertainty associated with the ABLH estimation,

$$
\sigma_{I 2}=\sqrt{\frac{1}{\sum_{i} 1 / \sigma_{i}^{2}}} .
$$

In Sect. 3, the ABLH inter-comparison is organised by pairs of instruments (e.g., AERI-DWL) taking account the incurred bias and the associated uncertainty. The bias between two instruments, $I_{1}$ and $I_{2}$, is defined

$$
\operatorname{bias}_{t_{h}}\left(I_{1}, I_{2}\right)=A B L H_{I_{1}}-A B L H_{I_{2}},
$$

and the associated uncertainty,

$$
\sigma_{\text {bias }_{t_{h}}}\left(I_{1}, I_{2}\right)=\sqrt{\sigma_{I_{1}}^{2}+\sigma_{I_{2}}^{2}}
$$

\section{DISCUSSION RESULTS}

\subsection{Case example I: clear air}

ABLH estimates from radar-EKF, DWL and AERI over the diurnal cycle of 16 April 2017 (Local time, LT = UTC - 5 h), are shown in Fig. 4 (sunrise, 1109 UTC; sunset, 0017 UTC 17 April). From 0700 to 1400 UTC, no thermal convection is apparent. The DWL ABLH estimates remain close to ground level, and the radar-EKF method yields ABLH estimates either lacking temporal continuity or partially locked to the RL. In this transition time - morning (TTM) regime, AERI-GM is giving more realistic results as it is the only ABLH method that takes the distinctive structure of the SBL into account.

At 1400 UTC, convection began (Fig. 4a). From this time forward, the ABLH estimates from EKF-radar and the DWL-VVSTD generally increased in concert until about 2300 UTC. However, disagreement grew between 1600 UTC and 1900 UTC, when clouds were present in the EZ (Fig. 4a). The maximum in radar reflectivity factor is expected at in the bottom of the $\mathrm{EZ}$ when clouds are present, while the maximum negative vertical gradient in radar reflectivity factor would be expected at the middle of the EZ in such conditions. On the other hand, the DWL-VVSTD estimated ABLH is based on the upper limit of turbulence, which is expected to coincide with the bottom of the EZ. Therefore, the EKF-radar-based ABLH estimate would be expected to be biased toward higher altitudes than the DWL-VVSTD-based ABLH estimate. From 2100 to 2300 UTC, differences in the ABLH estimates between these two methods can likely be explained as a result of using DWL-VVSTD estimates based on low-temporal resolution DWL data.

The AERI-PM consistently underestimates the ABLH by a factor of one-half or more (Fig. 4a). This unexpected result is still under study with the NOAA and Purdue partners of the VORTEX-Southeast project. 
(a)

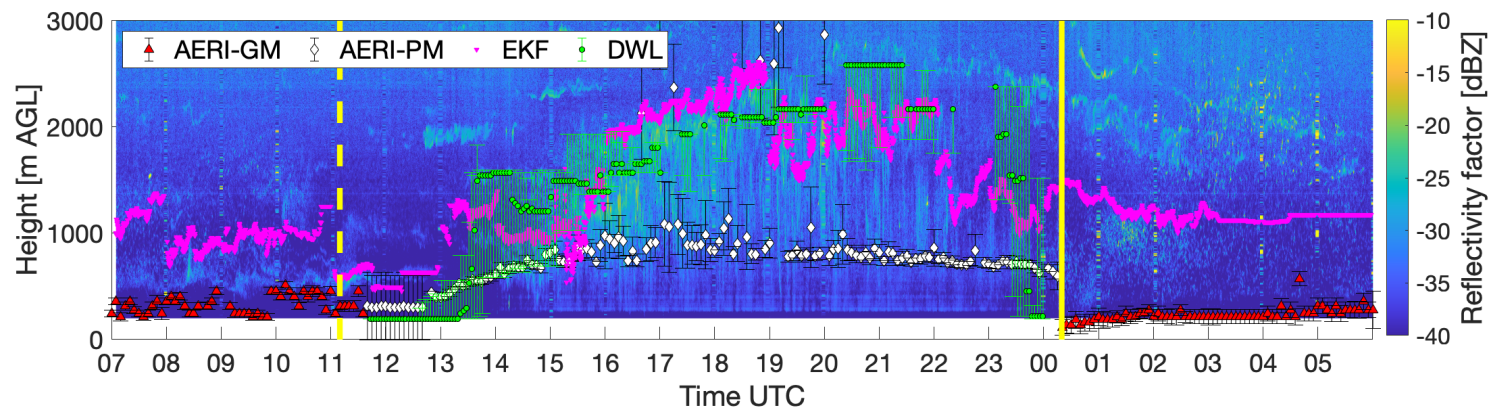

(b)

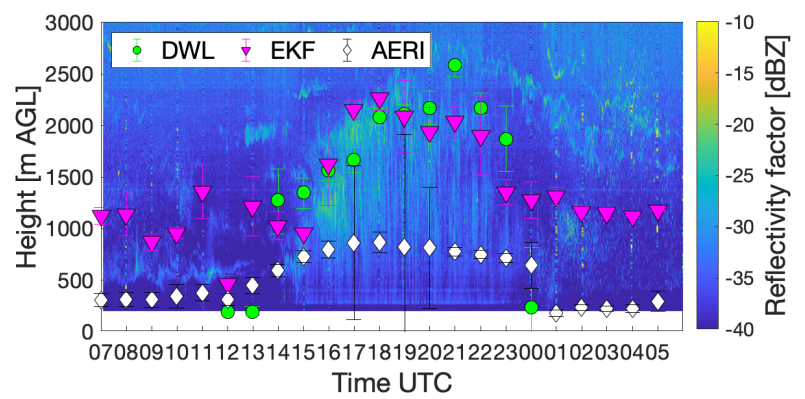

(c)

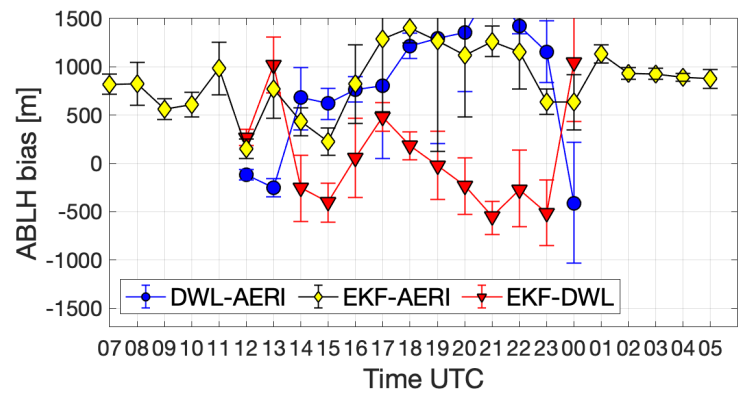

Figura 4. (a) ABLH estimated over the diurnal cycle of a clear day (0700 UTC 16 April 2017 to 0600 UTC 17 April 2017; LT = UTC-5h) from radar-EKF (magenta triangles), DWL-VVSTD (green dots), AERI PM (white diamonds) in the CBL regime between sunrise and sunset, and AERI GM in the SBL regime between sunset and runrise (red triangles). Also plotted are UMass S-band radar reflectivity factor (color fill), and times of local sunrise (yellow dashed line) and sunset (yellow solid line). (b) As in (a), but plotted as 1-h averages. Markers indicate the estimated value (Eq. 1); error bars indicate the $1 \sigma$ uncertainty (Eq. 2). (c) 1-h ABLH bias as function of time for DWL minus AERI (blue), EKF minus AERI (yellow), and EKF minus DWL (red). Markers indicate the estimated value (Eq. 5); error bars indicate the $1 \sigma$ uncertainty (Eq. 6).

The AERIoe profiles generally do not conform to the Ref. 1 CBL conceptual model on which this study is based. From the point of view of Information Theory, the largest amount of information used in the AERIoe retrieval is concentrated in the lower hundreds of meters of the atmosphere, and decreases exponentially with height. The reduced resolution of the AERioe temperature and moisture profiles above $1000 \mathrm{~m}$ may partly explain this poor ABLH retrieval. Owing to this substantial disagreement, we disregard this estimate hereafter.

\subsection{Case example II: cloudy air}

The case study presented in Fig. 5 (28 April 2017, 1330 - 2330 UTC; sunset, 1055 UTC; sunrise, 0027 UTC April 29) shows the ABLH estimations from the radar-EKF, the DWL-VVSTD, and AERI-GM compared to the RS reference during an episode in which ABLH was capped with clouds. Because entrainment occurs at the periphery of turbulent flow, the cloud base (particularly, in fair-weather cumulus) can be considered a proxy for the bottom of the EZ.

On one hand, the ABLHs estimated by the five RS available for this day are fairly coincident with the radarEKF ABLHs (Fig. 5a). As described in Sect. 2.1 and illustrated in Fig. 1, the EKF provides time-tracking estimates of the approximate EZ center height (CBL-to-FT infection point in Fig. 1) during the central daylight hours and maximum development of the CBL (1700-2100 UTC, 1200-1700 LT). In contrast, DWL ABLH are better estimates of the bottom of the EZ in these hours.

On the other hand, AERI ABLH estimates fall well below the other methods. We hypothesize that the cloud cover reduces the incoming infrared solar radiative flux, which affects the AERI radiance measurements and, consequently, the AERIoe-retrieved thermodynamic profiles. 
Fig. $5 \mathrm{c}$ shows relatively small biases (below $\pm 200 \mathrm{~m}$ ) between the radar-EKF and RS estimates during the convective time interval (1530-2300 UTC), whereas the DWL estimates are biased approximately $-400 \mathrm{~m}$ compared to RS. AERI ABLHs are largely underestimated for the reasons discussed earlire. From 1600-2000 UTC (Fig. 5c) the EKF-DWL bias and respective uncertainty is reduced, resulting in better agreement between both methods during the CBL period. Additionally, radar-EKF and DWL exhibit relatively small differences in relation to RS during this period (Fig. 5c).

(a)

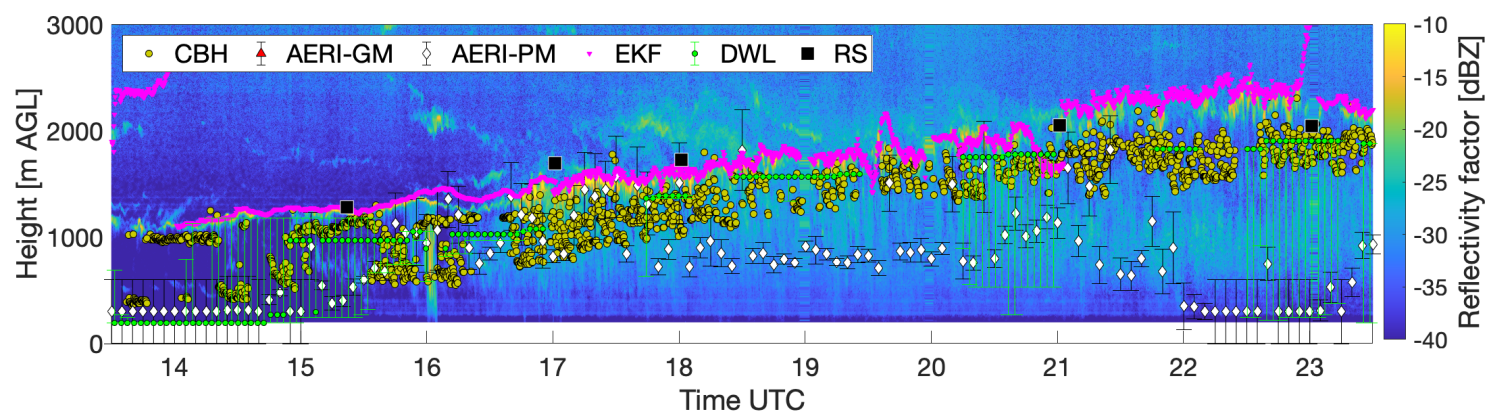

(b)

(c)
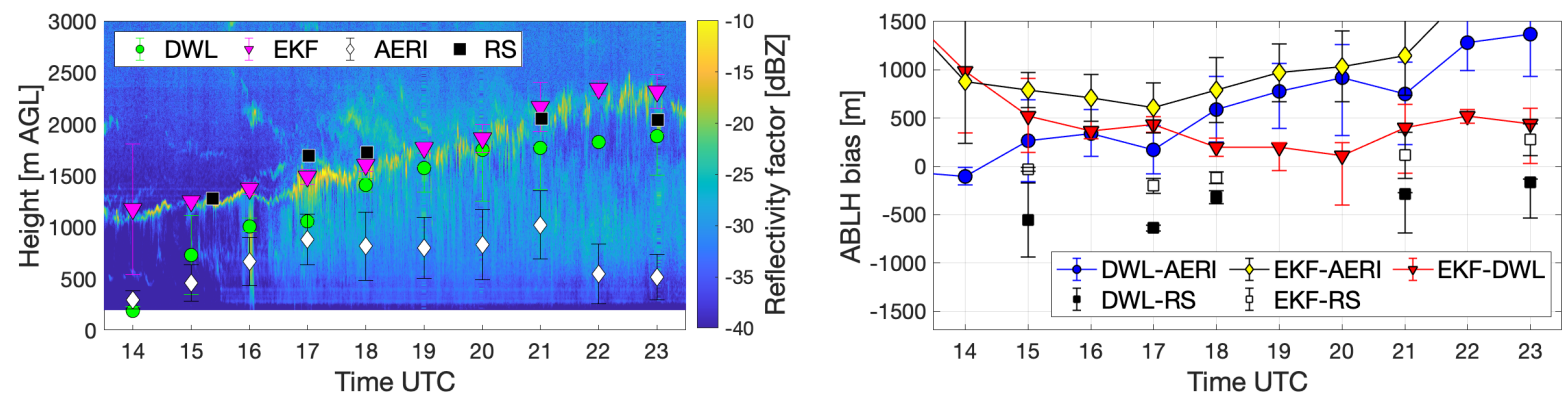

Figura 5. As in Fig. 4, but for an example of a cloudy afternoon (28 April 2017, 1330-2330 UTC). ABLH estimates from RS using the parcel method are plotted as black squares.

\section{CONCLUSIONS}

A synergistic multi-instrument remote-sensing approach was used to derive different estimates of the ABLH, combining S-band radar, DWL, and AERI observed ABL profiles. We applied an EKF to time series of vertical profiles of the radar reflectivity, a standard-deviation threshold method to DWL vertical (air) velocity profiles, and both the parcel method and the gradient method to virtual potential temperature profiles from AERI and radiosondes. The retrievals were compared over two different case days from VORTEX-SE 2017: a clear-sky day and a cloudy day.

In the SBL regime, the AERI-GM seems the most reliable method for estimating the ABLH, with uncertainty and bias performance superior to that of the DWL and the radar-EKF. In the CBL regime, the radar-EKF exhibited the best performance at tracking the EZ center height, while DWL-based ABLH estimates (near the bottom of the EZ) tracked closely and slightly below those from the radar-EKF. The AERIoe-based ABLH estimates were consistently too low, and were probably impacted by the change in infrared radiation in the presence of cloud cover.

Finally, synergistic remote sensing offers the advantages of balancing the drawbacks and strengths of the different instrument-method combinations (see Tab 2), formulating ALBH estimation schemes for different ABL regimes, and enabling the retrieval of more comprehensive, unambiguous ABLH estimates. 
Taula 2. Summary of strengths and weaknesses for each remote-sensing instrument and its associated ABLH retrieval methods.

\begin{tabular}{|c|c|c|c|}
\hline Instrument & Method & Strengths & Weaknesses \\
\hline $\begin{array}{l}\text { UMass S-band } \\
\text { radar }\end{array}$ & EKF & $\begin{array}{l}\text { - High temporal and spatial } \\
\text { resolution. } \\
\text { - Direct measurement of the } \\
\text { structure parameter for the } \\
\text { refractive index, } C_{n}^{2} \text {. } \\
\text { - Reliable ABLH estimation in } \\
\text { CBL regime. } \\
\text { - The erf-like model considers the } \\
\text { EZ middle as the ABLH. }\end{array}$ & $\begin{array}{l}\text { - Low signal at night and in } \\
\text { morning transition time. } \\
\text { - Detection of the RL in the } \\
\text { afternoon transition time. } \\
\text { - Very large files (1h-400 MB). }\end{array}$ \\
\hline \multirow[t]{2}{*}{$\begin{array}{l}\text { DWL (Halo } \\
\text { Stream Line XR) }\end{array}$} & $\begin{array}{l}\text { Threshold } \\
\text { method HR }\end{array}$ & $\begin{array}{l}\text { - Direct measurement of } \\
\text { turbulence. } \\
\text { - Low VVSTD threshold } \\
\text { sensitivity. } \\
\text { - Reliable ABLH estimation in } \\
\text { CBL regime. }\end{array}$ & $\begin{array}{l}\text { - Does not work in SBL. } \\
\text { - Very large files ( } 24 \mathrm{~h}-400 \mathrm{MB}) \text {. } \\
\text { - Height range limited by the } \\
\text { aerosol concentration. }\end{array}$ \\
\hline & $\begin{array}{l}\text { Threshold } \\
\text { method LR }\end{array}$ & $\begin{array}{l}\text { - Measurement of turbulence. } \\
\text { - Reliable ABLH estimation in } \\
\text { CBL regime. }\end{array}$ & $\begin{array}{l}\text { - High VVSTD threshold } \\
\text { sensitivity. } \\
\text { - Larger uncertainties than for } \\
\text { HR data. } \\
\text { - Does not work in SBL. } \\
\text { - Height range limited by the } \\
\text { aerosol concentration. }\end{array}$ \\
\hline \multirow[t]{2}{*}{$\begin{array}{l}\text { AERI (v4 } \\
\text { MP-3000A) }\end{array}$} & Parcel Method & $\begin{array}{l}\text { - } \text { Based on thermodynamic } \\
\text { properties of the ABL. }{ }^{1} \\
\text { - Reliable ABLH estimation in } \\
\text { TTM, but only when ABLH is } \\
\text { lower than } 1000 \mathrm{~m} .\end{array}$ & $\begin{array}{l}\text { - Low temporal and spatial } \\
\text { resolution. } \\
\text { - Degraded information content } \\
\text { and resolution at altitudes above } \\
1000 \mathrm{~m} \text {. } \\
\text { - Consistently underestimated } \\
\text { ABLH in CBL in this study. } \\
\text { - Unable to track the SBL height. }\end{array}$ \\
\hline & $\begin{array}{l}\text { Gradient Method } \\
\text { (SBL) }\end{array}$ & $\begin{array}{l}\text { - Measurement of thermodynamic } \\
\text { properties of the ABL. } \\
\text { - Able to track the SBL height. }\end{array}$ & $\begin{array}{l}\text { - Relatively low temporal and } \\
\text { spatial resolution. } \\
\text { - Degraded information content } \\
\text { and resolution at altitudes above } \\
1000 \mathrm{~m} \text {. }\end{array}$ \\
\hline
\end{tabular}




\section{Acknowledgements}

This work was supported by NOAA grants NA1501R4590232 and NA16OAR4590209, and by the Purdue University Dept. of Earth, Atmospheric, and Planetary Sciences (EAPS). CommSensLab-UPC (Excellence Unit MDM-2016-0600 funded by the Agencia Estatal de Investigación, Spain) collaborated via Spanish Gov. - EU Regional Development Funds, ARS project PGC2018-094132-B-I00 and ACTRIS-PPP project GA-739530. The Spanish National Science Foundation (Ministerio de Ciencia, Innovación y Universidades) funded doctoral grant PRE2018-086054 hold by M.P. Araujo da Silva. The University of Massachusetts, Microwave Remote Sensing Lab. and Purdue University, EAPS, deployed the mobile S-band radar, co-located Vaisala CL-31 ceilometer, and portable disdrometers. The NOAA National Severe Storms Laboratory deployed CLAMPS. The statements, findings, conclusions, and recommendations are those of the authors and do not necessarily reflect the views of the NOAA or the U.S. Department of Commerce.

\section{REFERENCES}

[1] Stull, R. B., [An Introduction to Boundary Layer Meteorology], Kluwer Academic Publishers (1988).

[2] Wallace, J. M. and Hobbs, P. V., [Atmospheric science: an introductory survey], vol. 92, Elsevier (2006).

[3] Koch, S. and Rasmussen, E. N., "VORTEX-SE: Program and Activities," 28th Conf. on Severe Local Storms, American Meteorological Society, Portland, Oregon (2016).

[4] Tanamachi, R. L., Frasier, S. J., Waldinger, J., LaFleur, A., Turner, D. D., and Rocadenbosch, F., "Progress toward Characterization of the Atmospheric Boundary Layer over Northern Alabama Using Observations by a Vertically Pointing, S-Band Profiling Radar during VORTEX-Southeast," Journal of Atmospheric and Oceanic Technology 36(11), 2221-2246 (2019).

[5] İnce, T., Frasier, S. J., Muschinski, A., and Pazmany, A. L., "An S-band frequency-modulated continuouswave boundary layer profiler: Description and initial results," Radio Science 38(4) (2003).

[6] Gossard, E. E., [Radar Research on the Atmospheric Boundary Layer], 477-527, American Meteorological Society (1990).

[7] Richter, J. H., "High Resolution Tropospheric Radar Sounding," Radio Science 4(12), 1261-1268 (1969).

[8] Schween, J. H., Hirsikko, A., Löhnert, U., and Crewell, S., "Mixing-layer height retrieval with ceilometer and Doppler lidar: from case studies to long-term assessment," Atmospheric Measurement Techniques $\mathbf{7}(11)$, 3685-3704 (2014).

[9] Berg, L. K., Newsom, R. K., and Turner, D. D., "Year-Long Vertical Velocity Statistics Derived from Doppler Lidar Data for the Continental Convective Boundary Layer," Journal of Applied Meteorology and Climatology 56, 2441-2454 (08 2017).

[10] Turner, D. D. and Löhnert, U., "Information Content and Uncertainties in Thermodynamic Profiles and Liquid Cloud Properties Retrieved from the Ground-Based Atmospheric Emitted Radiance Interferometer (AERI)," Journal of Applied Meteorology and Climatology 53(3), 752-771 (2014).

[11] Dirksen, R., Sommer, M., Immler, F., Hurst, D., Kivi, R., and Vömel, H., "Reference quality upper-air measurements: GRUAN data processing for the Vaisala RS92 radiosonde.," Atmospheric Measurement Techniques 7(12) (2014).

[12] Villalonga, J., Beveridge, S. L., Araujo da Silva, M. P., Tanamachi, R. L., Rocadenbosch, F., Turner, D. D., and Frasier, S. J., "Convective boundary-layer height estimation from combined radar and doppler lidar observations in vortex-se," (2020). Proc. SPIE 11531, Remote Sensing of Clouds and the Atmosphere XXV, 11531-32 (22 September); In print.

[13] Wagner, T. J., Klein, P. M., and Turner, D. D., "A new generation of ground-based mobile platforms for active and passive profiling of the boundary layer," Bulletin of the American Meteorological Society 100(1), 137-153 (2019).

[14] Contreras, R. F. and Frasier, S. J., "High-Resolution Observations of Insects in the Atmospheric Boundary Layer," Journal of Atmospheric and Oceanic Technology 25(12), 2176-2187 (2008).

[15] Lange, D., Rocadenbosch, F., Tiana-Alsina, J., and Frasier, S., "Atmospheric Boundary Layer Height Estimation Using a Kalman Filter and a Frequency-Modulated Continuous-Wave Radar," IEEE Transactions on Geoscience and Remote Sensing 53(6), 3338-3349 (2015). 
[16] Lange, D., Tiana-Alsina, J., Saeed, U., Tomás, S., and Rocadenbosch, F., "Atmospheric Boundary Layer Height Monitoring Using a Kalman Filter and Backscatter Lidar Returns," IEEE Transactions on Geoscience and Remote Sensing 52(8), 4717-4728 (2014).

[17] Browning, K. A. and Wexler, R., "The determination of kinematic properties of a wind field using doppler radar," Journal of Applied Meteorology 7(1), 105-113 (1968).

[18] Fujii, T. and Fukuchi, T., [Laser Remote Sensing], CRC Press (2005).

[19] Lenschow, D. H., Wulfmeyer, V., and Senff, C., "Measuring Second- through Fourth-Order Moments in Noisy Data," Journal of Atmospheric and Oceanic Technology 17(10), 1330-1347 (2000).

[20] Tucker, S. C., Senff, C. J., Weickmann, A. M., Brewer, W. A., Banta, R. M., Sandberg, S. P., Law, D. C., and Hardesty, R. M., "Doppler Lidar Estimation of Mixing Height Using Turbulence, Shear, and Aerosol Profiles," Journal of Atmospheric and Oceanic Technology 26(4), 673-688 (2009).

[21] Lenschow, D. H., Wyngaard, J. C., and Pennell, W. T., "Mean-field and second-moment budgets in a baroclinic, convective boundary layer," Journal of the Atmospheric Sciences 37(6), 1313-1326 (1980).

[22] Holzworth, G. C., "Estimates of mean maximum mixing depths in the contiguous United States," Monthly Weather Review 92(5), 235-242 (1964).

[23] Barlow, R. J., [Statistics: A Guide To The Use of Statistical Methods in the Physical Sciences], John Wiley \& sons (1989). 